\title{
Contralateral Suppression at Adrenal Venous Sampling Is Associated with Renal Impairment Following Adrenalectomy for Unilateral Primary Aldosteronism
}

\begin{abstract}
Ye Seul Yang ${ }^{1,2, *}$, Seung Hun Lee,*, Jung Hee Kim ${ }^{3}$, Jee Hee Yoo ${ }^{4}$, Jung Hyun Lee ${ }^{1}$, Seo Young Lee ${ }^{5}$ A Ram Hong ${ }^{6}$, Dong-Hwa Lee ${ }^{7}$ Jung-Min $\mathrm{Koh}^{3}$, Jae Hyeon $\mathrm{Kim}^{4}$, Sang Wan Kim ${ }^{1,8}$

${ }^{1}$ Department of Internal Medicine, Seoul National University College of Medicine, Seoul; ${ }^{2}$ Department of Internal Medicine, Uijeongbu St. Mary's Hospital, College of Medicine, The Catholic University of Korea, Uijeongbu; ${ }^{3}$ Division of Endocrinology and Metabolism, Department of Medicine, Asan Medical Center, University of Ulsan College of Medicine; ${ }^{4}$ Division of Endocrinology and Metabolism, Department of Medicine, Samsung Medical Center, Sungkyunkwan University School of Medicine, Seoul; ${ }^{5}$ Department of Internal Medicine, Mediplex Sejong Hospital, Incheon; ${ }^{6}$ Department of Internal Medicine, Chonnam National University Medical School, Gwangju; ${ }^{7}$ Department of Internal Medicine, Chungbuk National University Hospital, Cheongju; ${ }^{8}$ Division of Endocrinology and Metabolism, Seoul Metropolitan Government Seoul National University Boramae Medical Center, Seoul, Korea
\end{abstract}

Background: Adrenal venous sampling (AVS) is performed to distinguish the subtype of primary aldosteronism (PA). The clinical

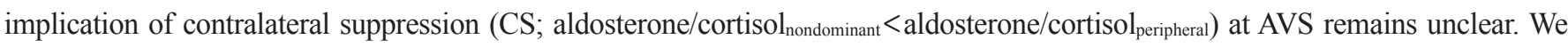
aimed to investigate the clinical significance of CS and its impact on postoperative outcomes after unilateral adrenalectomy.

Methods: In this retrospective observational multi-center study, we analyzed PA patients who underwent both successful adrenocorticotropin hormone-stimulated AVS and unilateral adrenalectomy. Successful cannulation was defined as the selectivity index (cortisol $\left._{\text {adrenal }} / \mathrm{cortisol}_{\text {peripheral }}\right) \geq 3$. Clinical and biochemical outcomes after unilateral adrenalectomy were evaluated based on the international Primary Aldosteronism Surgical Outcome consensus.

Results: Among 263 patients analyzed, 247 had CS (93.9\%). Patients with CS had lower serum potassium levels, higher plasma aldosterone concentration, higher aldosterone-to-renin ratio (ARR), and larger adenoma size than those without CS. Those with CS showed significantly higher lateralization index than those without CS $(P<0.001)$. Although postoperative blood pressure and ARR significantly decreased in those with CS, clinical and biochemical outcomes were comparable in both groups. When the cut-off value of age was determined using receiver operating characteristic (ROC) analysis, patients aged $\geq 50$ years old with contralateral sup-

Received: 25 March 2021, Revised: 21 June 2021, Accepted: 12 July 2021

Corresponding authors: Sang Wan Kim

Department of Internal Medicine, Seoul Metropolitan Government Seoul National University Boramae Medical Center, Seoul National University College of Medicine, 20 Boramae-ro 5-gil, Dongjak-gu, Seoul 07061, Korea Tel: +82-2-870-2223, Fax: +82-2-870-3863, E-mail: swkimmd@snu.ac.kr

Jae Hyeon Kim

Division of Endocrinology and Metabolism, Department of Medicine, Samsung

Medical Center, Sungkyunkwan University School of Medicine, 81 Irwon-ro, Gangnam-gu, Seoul 06351, Korea

Tel: +82-2-3410-1580, Fax: +82-2-3410-0393, E-mail: jaehyeon@skku.edu

\section{Copyright $(\odot) 2021$ Korean Endocrine Society}

This is an Open Access article distributed under the terms of the Creative Commons Attribution Non-Commercial License (https://creativecommons.org/ licenses/by-nc/4.0/) which permits unrestricted non-commercial use, distribution, and reproduction in any medium, provided the original work is properly cited.

*These authors contributed equally to this work. 
pression index (CSI; the ratio between aldosterone/cortisol nondominant $_{\text {and aldosterone/cortisol }}$ peripheral $) \leq 0.26$ had greater odds ratio (6.43; $95 \%$ confidence interval, 1.30 to 31.69$)$ of incident chronic kidney disease than those aged $<50$ years with CSI $>0.26$ after adjusting for other factors.

Conclusion: CS may not predict postoperative clinical and biochemical outcomes in subjects with unilateral aldosterone excess, but it is associated with postsurgical deterioration of renal function in subjects over 50 years with CSI $\leq 0.26$.

Keywords: Adrenalectomy; Glomerular filtration rate; Hyperaldosteronism; Hypertension; Phlebography; Treatment outcome

\section{INTRODUCTION}

Primary aldosteronism (PA) is the most common curable disease of secondary hypertension. Aldosterone production is inappropriately high, relatively independent of the renin-angiotensin-aldosterone system, and non-suppressible via sodium loading in PA [1,2]. The two major subtypes of PA are unilateral aldosterone-producing adenoma (APA) and bilateral adrenal hyperplasia (BAH). Subtype differentiation is a key step in the management of PA because treatment differs for these two subtypes - unilateral adrenalectomy is recommended for patients with unilateral APA, while medical treatment including mineralocorticoid receptor antagonist is recommended for patients with BAH [3].

Adrenal venous sampling (AVS) is the gold standard in determining the specific subtype. Therefore, the 2016 Endocrine Society guideline strongly recommends AVS when surgical treatment is feasible and desired by the patient [3]. However, a standardized protocol for AVS is still lacking. The lateralization in-

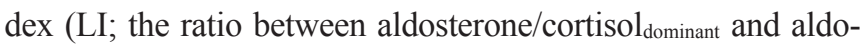
sterone/cortisol nondominant $_{\text {) }}$ is used to determine subtype of PA in most clinical centers. Although there is no consensus on the optimal LI cut-off in the subtype differentiation, LI $>4$ indicates unilateral PA and $3<\mathrm{LI}<4$ is a grey zone [4]. Some studies showed that LI predicts postoperative outcomes of hypertension $[5,6]$.

In some centers, contralateral suppression (CS; aldosterone/ cortisol $_{\text {nondominant }}<$ aldosterone/cortisol peripheral $_{\text {) }}$ together with LI is used to determine whether the subtype is unilateral PA [7]. However, the clinical significance of CS and its impact on postoperative outcomes after a unilateral adrenalectomy are still controversial $[6,8-10]$. Therefore we performed a retrospective study aimed to investigate whether the presence or degree of CS influences postoperative outcomes after adrenalectomy in unilateral PA.

\section{METHODS}

\section{Subject population}

In this multi-center retrospective study, we evaluated PA patients who underwent both successful adrenocorticotropin hormone (ACTH)-stimulated AVS and unilateral adrenalectomy. We enrolled 263 patients at Seoul National University Hospital (SNUH, $n=135$ ), Asan Medical Center (AMC, $n=93$ ), Samsung Medical Center (SMC, $n=26)$ and Seoul Metropolitan Government Boramae Medical Center (SMG-BMC, $n=9)$. Participants who had a follow-up period of less than 3 months after adrenalectomy were excluded. The present study was approved by the Institutional Review Board of each institute (Approval No.: SNUH and SMG-BMC, H-1801-010-911; AMC, 20160254; SMC, SMC 2018-02-055) and was conducted according to the Declaration of Helsinki. The need for informed consent was waived due to the retrospective nature of the study. Clinical data relating to PA patients at AMC were extracted using the Asan BiomedicaL research Environment, the de-identified clinical research data warehouse at the AMC [11], between May 2007 and April 2016.

\section{Assessment of anthropometric and biochemical parameters}

Data regarding age, sex, body mass index, blood pressure (BP), duration of hypertension, number and defined daily dose (DDD) of anti-hypertensive drugs taken, plasma renin activity (PRA), plasma aldosterone concentration (PAC), serum potassium levels, and serum creatinine levels were retrieved from the patients' medical records. Estimated glomerular filtration rate (GFR) was calculated with each patient's serum creatinine, age and sex using the Modification of Diet in Renal Disease formula: $175 \times$ serum creatinine (in $\mathrm{mg} / \mathrm{dL})[\exp (-1.154)] \times$ age (in years) $[\exp$ $(-0.203)] \times 0.742$ (if female). The initial BP was recorded at the first outpatient visit. Postoperative BP was measured once at the latest outpatient visit. Fasting blood samples ( 8 to 9 AM) were obtained as quickly as possible using a tourniquet with the pa- 
tient in the seated position. At SNUH, SMG-BMC, and AMC, PRA was measured by radioimmunoassay (RIA) using either renin RIA beads (before 2011; TFB Inc., Tokyo, Japan) or PRA RIA kit (after 2011; TFB Inc.), which had intra- and inter-assay coefficients of variations (CVs) of $3.8 \%$ and $6.7 \%$, respectively. Levels of PAC were determined by RIA using the SPAC-S aldosterone kit (TFB Inc.), which had intra- and inter-assay CVs of $4.7 \%$ and $4.5 \%$, respectively. PRA and PAC were measured by RIA using angiotensin I RIA kit (intra- and inter-assay CVs of $11.3 \%$ and $12.5 \%$ ) and aldosterone RIA kit (intra- and interassay CVs of $11.9 \%$ and 12.5\%) (Beckman Coulter, Prague, Czech Republic) at SMC.

\section{Diagnosis of PA}

Saline infusion test (SIT) was performed for diagnosis of PA in patients with hypertension who exhibited an increased plasma aldosterone-to-renin ratio (ARR) of $\geq 20$ and PAC of $>15 \mathrm{ng} / \mathrm{dL}$. A positive test was considered if PAC after SIT was $\geq 10 \mathrm{ng} / \mathrm{dL}$ [3]. Before confirming the diagnosis, all anti-hypertensive medications were changed to calcium channel blockers or alpha-adrenergic receptor blockers at least 4 weeks prior to measuring PAC and PRA, which do not interfere with the renin-angiotensin-aldosterone system. The subtype of PA was determined based on AVS results under ACTH stimulation. A successful AVS was defined as a selectivity index (i.e., the ratio of cortisol in the adrenal vein and the peripheral vein) of $\geq 3$. Unilateral PA was identified using cut-off values of $\mathrm{LI} \geq 4$, a potential indication for adrenalectomy. The presence of an adrenal adenoma was identified by the pathologists.

\section{Outcomes}

Clinical and biochemical outcomes after unilateral adrenalectomy were evaluated based on the international Primary Aldosteronism Surgical Outcome (PASO) consensus [12]. Complete clinical success was defined as the patient achieving a systolic BP of $<140 \mathrm{~mm} \mathrm{Hg}$ and a diastolic BP of $<90 \mathrm{~mm} \mathrm{Hg}$ without the use of anti-hypertensive medication. Partial clinical success was defined as unchanged BP with less anti-hypertensive medications or a reduction in BP with either the same or less amount of antihypertensive medication. Absent clinical success was defined as unchanged or increased BP with either the same amount or an increase in antihypertensive medication. Unchanged BP levels defined as a difference (preoperatively vs. postoperatively) in systolic BP of $<20 \mathrm{~mm} \mathrm{Hg}$ and diastolic BP of $<10 \mathrm{~mm} \mathrm{Hg}$; reduction or increase in BP defined as a difference in systolic $\mathrm{BP}$ of $\geq 20 \mathrm{~mm} \mathrm{Hg}$ or diastolic BP of $\geq 10 \mathrm{~mm} \mathrm{Hg}$, or both. An- tihypertensive medication was expressed as DDD assessed by Anatomical Therapeutic Chemical/Defined Daily Dose (ATC/ DDD) index 2019. Unchanged, less and increased anti-hypertensive medication were defined as a change of the DDD between pre-adrenalectomy and post-adrenalectomy; decrease or increase less than 0.5 times, a decrease of $\geq 0.5$ times and an increase of $\geq 0.5$ times, respectively. The number of medications was represented by the number of classes of antihypertensive medications.

Complete biochemical success was defined as the correction of hypokalemia and normalization of the ARR (postoperative ARR $<20$ ng/dL per ng/mL/hr). Partial biochemical success was defined as the correction of hypokalemia and a raised ARR with $\geq 50 \%$ decrease in baseline PAC. Absent biochemical success was defined as persistent hypokalemia or persistent raised ARR. Hypokalemia was defined as serum potassium levels of $<3.5 \mathrm{mmol} / \mathrm{L}$ and normalization of hypokalemia was defined as serum potassium levels of $\geq 3.5 \mathrm{mmol} / \mathrm{L}$ after treatment if present at pre-adrenalectomy. Postoperative hyperkalemia was defined if the serum potassium level was above $5.5 \mathrm{mmol} / \mathrm{L}$ at 3 months after surgery.

For assessing renal outcomes, we defined chronic kidney disease (CKD) as GFR $<60 \mathrm{~mL} / \mathrm{min} / 1.73 \mathrm{~m}^{2}$. Incident $\mathrm{CKD}$ was defined when the postoperative GFR was less than $60 \mathrm{~mL} / \mathrm{min} /$ $1.73 \mathrm{~m}^{2}$ in the case of preoperative GFR was $\geq 60 \mathrm{~mL} / \mathrm{min} / 1.73 \mathrm{~m}^{2}$ and an overall decline in GFR was $\geq 15 \mathrm{~mL} / \mathrm{min} / 1.73 \mathrm{~m}^{2}$ from the preoperative GFR [13]. Cardiovascular diseases included coronary artery diseases, myocardial infarction, and congestive heart failure. Cerebrovascular diseases included ischemic or hemorrhagic stroke [14].

\section{Statistical analyses}

Data are presented as mean \pm standard deviation, or in the case of skewed distributions, as median (interquartile range [IQR]). The independent-samples $t$ test was used to compare two groups, and the Mann-Whitney $U$ test was used in the case of skewed distributions. The chi-square test and Fisher's exact tests were used to compare the categorical variables. Difference within each group was analyzed using paired $t$ test. Logistic regression models were performed to evaluate the odds ratios (OR) with $95 \%$ confidence intervals (95\% CIs) of preoperative clinical and biochemical parameters for postoperative outcomes. Multivariate logistic regression models with stepwise backward methods were performed including the variables a $P$ value of $<0.05$ in the univariate analysis. All statistical analyses were performed using SPSS software version 21 (IBM Co., Armonk, 
Table 1. Baseline (Preadrenalectomy) Characteristics of Patients with Unilateral Primary Aldosteronism

\begin{tabular}{|c|c|c|c|}
\hline Variable & $\begin{array}{l}\text { Contralateral suppression }(+) \\
\qquad(n=247)\end{array}$ & $\begin{array}{l}\text { Contralateral suppression }(-) \\
\qquad(n=16)\end{array}$ & $P$ value \\
\hline Number & $247(93.9)$ & $16(6.1)$ & \\
\hline Male sex & $128(50.2)$ & $4(25.0)$ & 0.038 \\
\hline Age at diagnosis, yr & $50.0 \pm 10.6$ & $50.5 \pm 11.0$ & 0.866 \\
\hline BMI, $\mathrm{kg} / \mathrm{m}^{2}$ & $24.7 \pm 3.6$ & $22.9 \pm 2.7$ & 0.044 \\
\hline Systolic BP, mm Hg & $144.6 \pm 19.3$ & $140.9 \pm 15.6$ & 0.448 \\
\hline Diastolic BP, mm Hg & $90.8 \pm 13.6$ & $85.9 \pm 10.0$ & 0.160 \\
\hline Serum potassium, $\mathrm{mmol} / \mathrm{L}$ & $3.2 \pm 0.6$ & $3.6 \pm 0.5$ & 0.004 \\
\hline Hypokalemia & $168(68.3)$ & $7(43.8)$ & 0.043 \\
\hline Serum creatinine, $\mathrm{mg} / \mathrm{dL}$ & $0.89 \pm 0.27$ & $0.81 \pm 0.25$ & 0.234 \\
\hline GFR, $\mathrm{mL} / \mathrm{min} / 1.73 \mathrm{~m}^{2}$ & $85.0 \pm 21.7$ & $87.4 \pm 20.4$ & 0.672 \\
\hline $\mathrm{GFR}<60 \mathrm{~mL} / \mathrm{min} / 1.73 \mathrm{~m}^{2}$ & $29(11.6)$ & $2(11.1)$ & 1.000 \\
\hline $\mathrm{PAC}, \mathrm{ng} / \mathrm{dL}$ & $39.5(28.4-55.9)$ & $28.3(21.0-54.8)$ & 0.017 \\
\hline PRA, ng/mL/hr & $0.20(0.10-0.34)$ & $0.27(0.16-0.52)$ & 0.959 \\
\hline ARR, ng/dL per ng/mL/hr & $287.7 \pm 268.1$ & $144.7 \pm 97.7$ & $<0.001$ \\
\hline PAC after SIT, ng/dL & $29.6(19.3-44.0)$ & $18.4(14.7-26.3)$ & 0.026 \\
\hline Lateralization index during AVS & $23.0(12.0-43.5)$ & $6.1(5.2-7.6)$ & $<0.001$ \\
\hline Mass size on $\mathrm{CT}, \mathrm{cm}$ & $1.5(1.3-1.9)$ & $1.2(1.0-2.2)$ & 0.030 \\
\hline Hypertension & $242(98.0)$ & $16(100.0)$ & 1.000 \\
\hline Duration of hypertension, yr & $6.0(2.0-10.0)$ & $4.5(1.0-15.0)$ & 0.463 \\
\hline No. of antihypertensive drugs & $2.0(1.0-3.0)$ & $2.0(2.0-3.0)$ & 0.812 \\
\hline DDD of antihypertensive drugs & $2.55 \pm 1.88$ & $2.29 \pm 1.46$ & 0.593 \\
\hline Diabetes & $36(14.6)$ & $3(20.0)$ & 0.474 \\
\hline Cardiovascular disease & $27(10.9)$ & $2(12.5)$ & 0.692 \\
\hline Cerebrovascular disease & $19(7.7)$ & $1(6.3)$ & 1.000 \\
\hline
\end{tabular}

Values are expressed as number (\%), mean \pm standard deviation, or median (interquartile range).

BMI, body mass index; BP, blood pressure; GFR, glomerular filtration rate; PAC, plasma aldosterone concentration; PRA, plasma renin activity; ARR, aldosterone-to-renin ratio; SIT, saline infusion test; AVS, adrenal venous sampling; CT, computed tomography; DDD, defined daily dose.

NY, USA), and differences with a $P$ value of $<0.05$ were considered statistically significant.

\section{RESULTS}

Among the 263 enrolled patients, 247 (93.9\%) had CS. Compared to those without $\mathrm{CS}$, patients with $\mathrm{CS}$ had lower serum potassium levels $(P=0.004)$, higher PAC $(P=0.017)$, higher ARR $(P<0.001)$, higher PAC after SIT $(P=0.026)$, and larger adenoma size on computed tomography scan before adrenalectomy ( $P=0.030$ ) (Table 1$)$. Patients with CS showed significantly higher LI than those without CS (median, 23.0 [IQR, 12.0 to 43.5 ] vs. 6.1 [IQR, 5.2 to 7.6], $P<0.001$ ) (Fig. 1). Meanwhile, no significant difference in the severity and duration of hypertension, renal function and presence of comorbidity was noted between two groups.

The postoperative outcomes of subjects with and without CS are shown in Table 2. More than half $(56.0 \%)$ of the patients with CS and $43.8 \%$ of the patients without CS showed complete clinical success. The decrease in systolic BP and diastolic BP was more significant in patients with CS than those without CS. Subject with CS tended to have higher complete or partial improvement in clinical outcomes assessed by PASO study than those without CS $(P=0.054)$. The DDD and number of antihypertensive drugs were comparable between the two groups also.

Almost all patients in the two groups exhibited normalization of hypokalemia. After adrenalectomy, the decrease in PAC and ARR was more profound in patients with CS than those without 


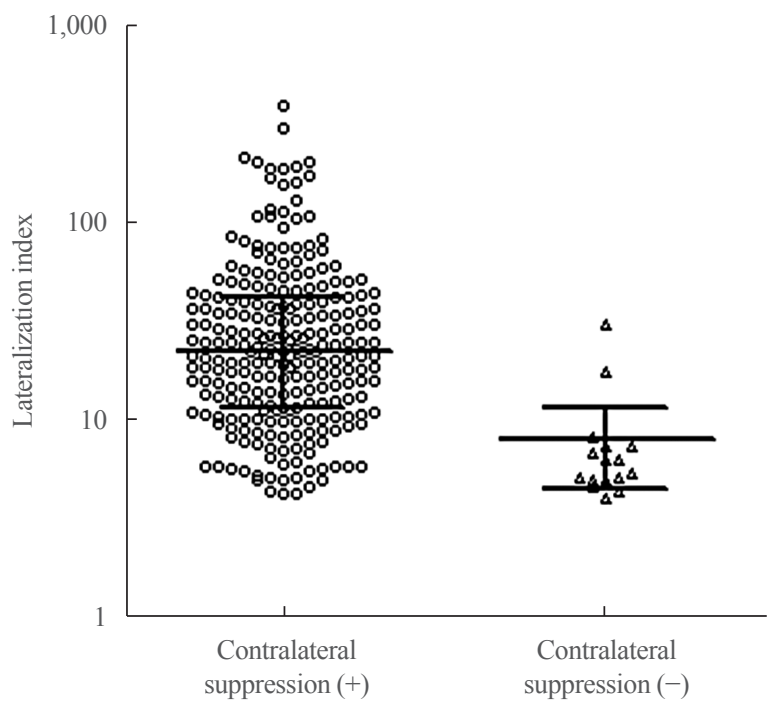

Fig. 1. Lateralization index between patients with and without contralateral suppression. Data are median with interquartile range. Using a Mann-Whitney $U$ test, $P<0.001$.

CS. However, biochemical outcomes according to the PASO study were not different according to the CS.

The median follow-up time for renal function was 380 days. Patients with CS had higher serum creatinine $(P=0.002)$ and lower GFR $(P=0.003)$ than those without CS after adrenalectomy (Table 2), even though the serum creatinine and GFR were not significantly different between two groups before adrenalectomy $(P=0.234$ and $P=0.672)$ (Table 1$)$. It seemed that the renal deteriorations (increase of serum creatinine levels and decrease of GFR) were more prominent in patients with CS. The GFR significantly decreased after adrenalectomy in patients with CS ( $n=215$ of $247,84.8 \pm 21.8$ to $69.6 \pm 21.7, P<0.001$ ), but not in patients without CS ( $n=14$ of $16,88.5 \pm 19.7$ to $85.6 \pm$ 19.1, $P=0.388$ ) in PA patients who had the data of GFR both before and after adrenalectomy (Fig. 2).

Associations of several clinical and biochemical parameters as predictive factors for clinical and biochemical outcomes were evaluated by logistic regression analysis. The cut-off value of contralateral suppression index (CSI; the ratio between aldosterone/cortisol $l_{\text {nondominant }}$ and aldosterone/cortisol $\left.l_{\text {peripheral }}\right)$ and LI were determined using receiver operating characteristic (ROC) analysis. LI >12 (OR, 2.57; 95\% CI, 1.34 to 4.91 ), duration of hypertension (OR, $0.92 ; 95 \% \mathrm{CI}, 0.87$ to 0.97 ), and DDD of antihypertensive drugs (OR, $0.66 ; 95 \% \mathrm{CI}, 0.55$ to 0.80 ) predicted postoperative complete clinical success. CS showed an association with clinical improvement, which included complete clinical success and partial clinical success. However, after adjust- ment with lateralization, systolic BP, duration of hypertension and DDD of antihypertensive drugs, CS lost its significant association (Supplemental Table S1).

We analyzed the associations of several clinical and biochemical parameters as predictive factors for the incident CKD using logistic regression models. CSI (OR, 0.73; 95\% CI, 0.59 to 0.91 ), LI (OR, 1.01; 95\% CI, 1.00 to 1.01), age of onset (OR, $1.04 ; 95 \%$ CI, 1.01 to 1.07 ), preoperative GFR (OR, 0.98; $95 \%$ CI, 0.96 to 1.00 ), and hypokalemia (OR, $4.12 ; 95 \%$ CI, 1.54 to 11.0) predicted postoperative incident CKD. Postoperative incident CKD was significantly associated with the CSI after adjusting for other factors (Table 3).

We performed ROC analysis to determine the cut-off value of parameters associated to the renal impairment. Area under the curve (AUC) for CSI, LI, and age of onset was 0.65 (95\% CI, 0.57 to 0.74$), 0.59$ ( $95 \% \mathrm{CI}, 0.50$ to 0.69$)$, and 0.61 (95\% CI, 0.53 to 0.70 ), respectively (Fig. 3). Patients aged $\geq 50$ years old with CSI $\leq 0.26$ had greater odds (OR, 9.18; 95\% CI, 2.03 to 41.52) of incident CKD than those aged $<50$ years with CSI $>0.26$. The OR was 6.43 (95\% CI, 1.30 to 31.69$)$ after adjusting for LI, preoperative GFR and duration of hypertension (Table 4).

\section{DISCUSSION}

In this multi-center retrospective study of 263 PA patients who underwent both AVS and unilateral adrenalectomy, those with $\mathrm{CS}$ at AVS did not show significant clinical and biochemical success compared with those without CS after adrenalectomy when assessed by PASO study criteria. CS was not an independent or better predictor of clinical improvement after adrenalectomy compared to LI. However, the CSI was associated with postoperative renal impairment in patients with unilateral PA especially over 50 years old, while LI was not. To the best of our knowledge, the present study provided the first finding that CSI was associated with the renal impairment after adrenalectomy in unilateral PA.

Postoperative GFR was significantly associated with CSI (the more suppressed in the contralateral gland, the lower the GFR), and its association was significant after adjusting for preoperative GFR in the present study. Only a few studies on renal function after adrenalectomy according to the presence or absence of CS have been conducted. One study [8] showed that postoperative urinary albumin to creatinine ratio was significantly lower in those with CS, but data on the albumin to creatinine ratio were available in only $42.5 \%$ of patients in that study, and the median reduction was not significantly different in both 
Table 2. Postoperative Outcomes of Patients with Unilateral Primary Aldosteronism

\begin{tabular}{|c|c|c|c|}
\hline Variable & $\begin{array}{l}\text { Contralateral suppression }(+) \\
\qquad(n=247)\end{array}$ & $\begin{array}{l}\text { Contralateral suppression }(-) \\
\qquad(n=16)\end{array}$ & $P$ value \\
\hline \multicolumn{4}{|l|}{ Clinical outcomes } \\
\hline Clinical outcomes by PASO study & & & 0.100 \\
\hline Complete clinical success & $136(56.0)$ & $7(43.7)$ & \\
\hline Partial clinical success & $76(31.3)$ & $4(25.0)$ & \\
\hline Absent clinical success & $31(12.7)$ & $5(31.3)$ & \\
\hline Complete or partial clinical success & $212(87.2)$ & $11(68.8)$ & 0.054 \\
\hline $\mathrm{SBP}, \mathrm{mm} \mathrm{Hg}$ & $129.4 \pm 14.0$ & $136.6 \pm 18.2$ & 0.060 \\
\hline DBP, mm Hg & $82.7 \pm 9.6$ & $87.5 \pm 13.1$ & 0.066 \\
\hline$\Delta \mathrm{SBP}, \mathrm{mm} \mathrm{Hg}$ & $-15.2 \pm 22.3$ & $-3.5 \pm 16.2$ & 0.047 \\
\hline$\triangle \mathrm{DBP}, \mathrm{mm} \mathrm{Hg}$ & $-8.1 \pm 15.0$ & $2.7 \pm 11.2$ & 0.007 \\
\hline High $\mathrm{BP}(\mathrm{SBP}>140$ and $\mathrm{DBP}>90)$ & $21(8.6)$ & $5(31.3)$ & 0.014 \\
\hline No. of antihypertensive drugs & $0(0$ to 1$)$ & $1(0$ to 2$)$ & 0.110 \\
\hline DDD of antihypertensive drugs & $0.00(0.00-1.00)$ & $0.75(0.00-2.00)$ & 0.165 \\
\hline$\Delta$ No. of antihypertensive drugs & $-1(-2$ to -1$)$ & $-1(-2$ to 0$)$ & 0.517 \\
\hline$\Delta \mathrm{DDD}$ of antihypertensive drugs & $-1.86 \pm 1.81$ & $-1.31 \pm 1.59$ & 0.233 \\
\hline \multicolumn{4}{|l|}{ Biochemical outcomes } \\
\hline Biochemical outcomes by PASO study & & & 0.624 \\
\hline Complete biochemical success & $175(74.8)$ & $10(71.4)$ & \\
\hline Partial biochemical success & $38(16.2)$ & $2(14.3)$ & \\
\hline Absent biochemical success & $21(9.0)$ & $2(14.3)$ & \\
\hline Complete or partial biochemical success & $213(91.0)$ & $12(85.7)$ & 0.626 \\
\hline Normalization of hypokalemia & $167 / 168(99.4)$ & $7 / 7(100)$ & 1.000 \\
\hline Postoperative hyperkalemia & $8(3.3)$ & 0 & 1.000 \\
\hline $\mathrm{PAC}, \mathrm{ng} / \mathrm{dL}$ & $12.0(7.8$ to 17.2$)$ & $12.8(8.4$ to 20.2$)$ & 0.802 \\
\hline $\mathrm{PRA}, \mathrm{ng} / \mathrm{mL} / \mathrm{hr}$ & $1.30(0.52$ to 2.50$)$ & $1.30(0.22$ to 5.02$)$ & 0.610 \\
\hline ARR, ng/dL per ng/mL/hr & $10.3(4.8$ to 21.8$)$ & $9.5(2.9$ to 27.3$)$ & 0.746 \\
\hline$\Delta \mathrm{PAC}, \mathrm{ng} / \mathrm{dL}$ & $-27.5(-42.4$ to -13.8$)$ & $-11.4(-20.0$ to -6.9$)$ & 0.005 \\
\hline$\Delta \mathrm{PRA}, \mathrm{ng} / \mathrm{mL} / \mathrm{hr}$ & $0.80(0.26$ to 2.32$)$ & 1.03 (0.09 to 4.57$)$ & 0.673 \\
\hline$\Delta \mathrm{ARR}, \mathrm{ng} / \mathrm{dL}$ per ng/mL/hr & $-200.8(-381.3$ to -72.4$)$ & $-92.2(-153.9$ to -36.4$)$ & 0.016 \\
\hline $\mathrm{PAC}$ reduction, $\%$ & $72.3(52.2$ to 82.4$)$ & $47.0(23.3$ to 75.1$)$ & 0.028 \\
\hline \multicolumn{4}{|l|}{ Renal function } \\
\hline Serum creatinine, $\mathrm{mg} / \mathrm{dL}$ & $1.10 \pm 0.44$ & $0.82 \pm 0.25$ & 0.002 \\
\hline $\mathrm{GFR}, \mathrm{mL} / \mathrm{min} / 1.73 \mathrm{~m}^{2}$ & $69.7 \pm 21.7$ & $85.6 \pm 19.1$ & 0.003 \\
\hline$\Delta$ Serum creatinine, $\mathrm{mg} / \mathrm{dL}$ & $0.21 \pm 0.29$ & $0.03 \pm 0.08$ & 0.001 \\
\hline$\Delta \mathrm{GFR}, \mathrm{mL} / \mathrm{min} / 1.73 \mathrm{~m}^{2}$ & $-15.2 \pm 18.0$ & $-2.9 \pm 10.8$ & 0.003 \\
\hline GFR $<60$ & $68(31.3)$ & $1(7.1)$ & 0.070 \\
\hline
\end{tabular}

Values are expressed as number (\%), mean \pm standard deviation, or median (interquartile range).

PASO, Primary Aldosteronism Surgery Outcome; SBP, systolic blood pressure; DBP, diastolic blood pressure; BP, blood pressure; DDD, defined daily dose; PAC, plasma aldosterone concentration; PRA, plasma renin activity; ARR, aldosterone-to-renin ratio; GFR, glomerular filtration rate.

groups. It has been known that excess aldosterone can lead to a glomerular hyperfiltration injury secondary to renal vascular re- modeling [15]. The more correction of hyperaldosteronism in those with lower CSI may lead to a stronger reduction in the rel- 


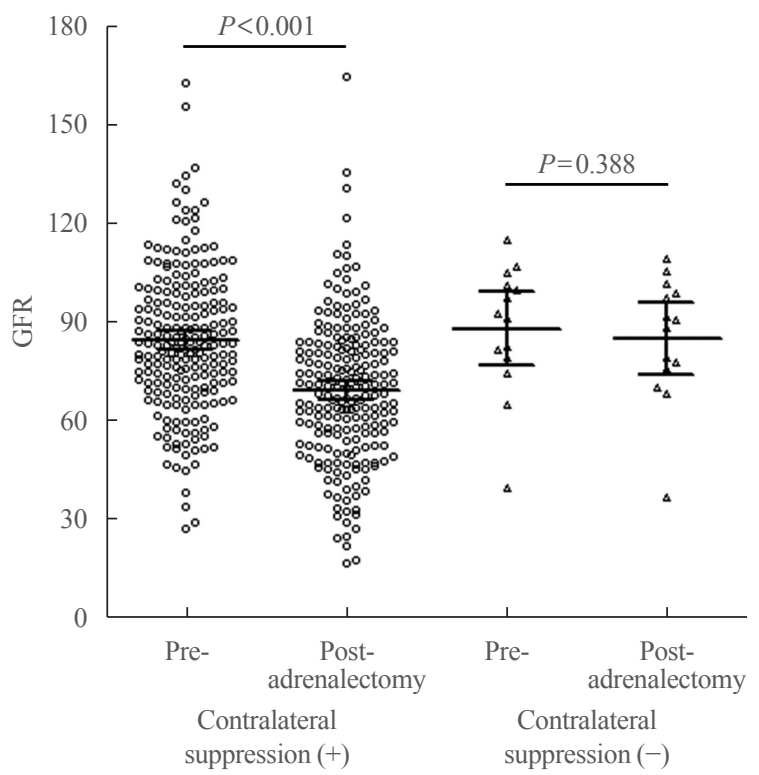

Fig. 2. Changes of glomerular filtration rate after adrenalectomy according to the presence or absence of contralateral suppression. Data are mean with $95 \%$ confidence intervals. A decrease of glomerular filtration rate (GFR) after adrenalectomy within each group was analyzed using paired $t$ test.

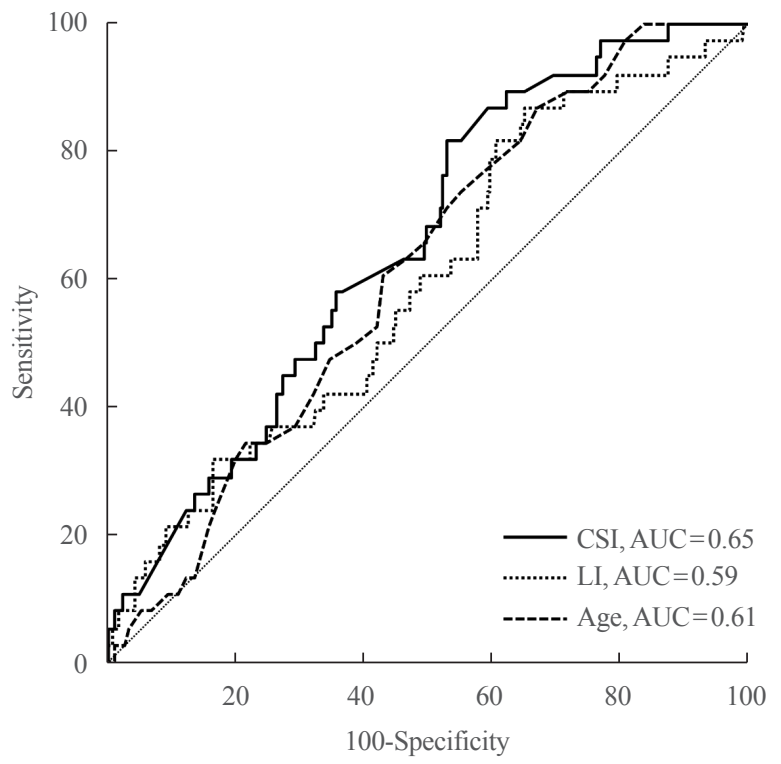

Fig. 3. Receiver operating characteristic curves of contralateral suppression index (CSI), lateralization index (LI), and age as predictors of incident chronic kidney disease after adrenalectomy. AUC, area under the curve.

ative glomerular hyperfiltration, as proposed by Sechi et al.'s studies $[16,17]$. On the other hand, our previous study showed that surgical and medical treatment provided similar ameliora-
Table 3. Odds Ratios of Preoperative Clinical and Biochemical Parameters for Postoperative Incident CKD in Patients with Unilateral Primary Aldosteronism

\begin{tabular}{|c|c|c|}
\hline Variable & $\begin{array}{c}\text { OR } \\
(95 \% \mathrm{CI})\end{array}$ & $\begin{array}{l}\text { Adjusted OR } \\
(95 \% \mathrm{CI})\end{array}$ \\
\hline $\begin{array}{l}\text { Contralateral suppression } \\
\text { index, } / 0.1 \text { unit }\end{array}$ & $0.73(0.59-0.91)$ & $0.78(0.62-0.99)$ \\
\hline Lateralization index, /1 unit & $1.01(1.00-1.01)$ & $1.00(1.00-1.01)$ \\
\hline Mass size on $\mathrm{CT}, / \mathrm{mm}$ & $1.05(1.00-1.10)$ & \\
\hline Age of onset, /yr & $1.04(1.00-1.07)$ & $1.04(1.00-1.08)$ \\
\hline Systolic BP, /mm Hg & $1.01(0.99-1.03)$ & \\
\hline Duration of hypertension, /yr & $1.05(1.00-1.10)$ & \\
\hline $\mathrm{GFR}, / \mathrm{mL} / \mathrm{min} / 1.73 \mathrm{~m}^{2}$ & $0.98(0.96-1.00)$ & $1.00(0.97-1.01)$ \\
\hline Hypokalemia & $4.12(1.54-11.0)$ & $3.46(1.24-9.64)$ \\
\hline $\mathrm{PAC}, / \mathrm{ng} / \mathrm{dL}$ & $1.01(1.00-1.02)$ & \\
\hline $\mathrm{PRA}, / \mathrm{ng} / \mathrm{mL} / \mathrm{hr}$ & $1.15(0.95-1.39)$ & \\
\hline $\mathrm{ARR}, / \mathrm{ng} / \mathrm{dL}$ per ng/mL/hr & $1.00(1.00-1.00)$ & \\
\hline $\mathrm{PAC}$ after SIT, /ng/dL & $1.01(1.00-1.02)$ & \\
\hline $\begin{array}{l}\text { DDD of antihypertensive } \\
\text { drugs, } / 1 \text { unit }\end{array}$ & $1.10(0.93-1.31)$ & \\
\hline
\end{tabular}

Incident $\mathrm{CKD}$ was defined when the postoperative GFR was less than $60 \mathrm{~mL} / \mathrm{min} / 1.73 \mathrm{~m}^{2}$ in the case of preoperative GFR was $\geq 60 \mathrm{~mL} /$ $\min / 1.73 \mathrm{~m}^{2}$ and an overall decline in GFR was $\geq 15 \mathrm{~mL} / \mathrm{min} / 1.73 \mathrm{~m}^{2}$ from the preoperative GFR.

CKD, chronic kidney disease; OR, odds ratio; CI, confidence interval; CT, computed tomography; BP, blood pressure; GFR, glomerular filtration rate; PAC, plasma aldosterone concentration; PRA, plasma renin activity; ARR, aldosterone-to-renin ratio; SIT, saline infusion test; DDD, defined daily dose.

Table 4. Odds Ratios for Postoperative Incident CKD in Patients with Unilateral Primary Aldosteronism According to Age and Contralateral Suppression Index

\begin{tabular}{lcc}
\hline & $\begin{array}{c}\text { OR } \\
(95 \% \mathrm{CI})\end{array}$ & $\begin{array}{c}\text { Adjusted OR } \\
(95 \% \mathrm{CI})^{\mathrm{a}}\end{array}$ \\
\hline Age $<50 \mathrm{yr}$ and CSI $>0.26$ & 1.00 & 1.00 \\
Age $\geq 50$ yr and CSI $>0.26$ & $2.50(0.46-13.56)$ & $2.16(0.38-12.21)$ \\
Age $<50$ yr and CSI $\leq 0.26$ & $4.76(1.00-22.62)$ & $4.01(0.83-19.40)$ \\
Age $\geq 50$ yr and CSI $\leq 0.26$ & $9.18(2.03-41.52)$ & $6.43(1.30-31.69)$ \\
\hline
\end{tabular}

Incident $\mathrm{CKD}$ was defined when the postoperative glomerular filtration rate (GFR) was less than $60 \mathrm{~mL} / \mathrm{min} / 1.73 \mathrm{~m}^{2}$ in the case of preoperative GFR was $\geq 60 \mathrm{~mL} / \mathrm{min} / 1.73 \mathrm{~m}^{2}$ and an overall decline in GFR was $\geq 15$ $\mathrm{mL} / \mathrm{min} / 1.73 \mathrm{~m}^{2}$ from the preoperative GFR.

CKD, chronic kidney disease; OR, odds ratio; CI, confidence interval; CSI, contralateral suppression index.

${ }^{a}$ Lateralization index, preoperative GFR and duration of hypertension were adjusted. 
tion of hypokalemia and hypertension; however, there was a greater decrease in GFR and higher serum potassium levels in the surgical treatment group than in the medical treatment group in patients with PA who were aged $>60$ years [18]. Another retrospective study showed that the GFR declined in the 6 months after the treatments - both adrenalectomy and medical treatment-however, long-term annual decline in GFR and risk for incident CKD were not significantly different between the patients with unilateral adrenalectomy and those with essential hypertension patient [13]. Therefore, it will be interesting to closely observe whether GFR reduction following surgery persists in patients with CS.

Additionally, in our study, postoperative hyperkalemia occurred only in patients with CS (8/247, 3.3\%). In our previous study, impaired preoperative renal function was associated with prolonged postoperative hyperkalemia in patients with APA [19]. Shariq et al. [20] have reported that a CSI of $<0.47$ was predictive for postoperative hyperkalemia. Post-adrenalectomy hyperkalemia in patients with unilateral PA results from the insufficiency of aldosterone secretion in the remaining adrenal gland immediately after surgery [19,21]. Similar to the cases of hyperkalemia, GFR reduction in patients with CS may be related to post-adrenalectomy hypoaldosteronism that may result in insufficient renal perfusion.

In our study, those with CS showed more definite features of PA (lower serum potassium level, higher ARR, and higher PAC after SIT) than those without CS. These clinical differences between the two groups suggest that CS is more likely to occur when disease activity (e.g., autonomous unilateral aldosterone production) of PA is more severe as previously suggested [10]. CS means that the activity of the contralateral gland is decreased. By contrast, the absence of CS suggests that a contralateral gland might cause persistent aldosterone production and result in a poorer BP outcome or recurrence after unilateral adrenalectomy as in the case of "one-side dominant" bilateral PA [22]. Therefore, even though clinical characteristics at pre-adrenalectomy were shown to be more severe in the case of CS, hypertension improvement and the degree of reduction of ARR could be greater in those with CS after adrenalectomy.

In the present study, CS itself may be associated with postoperative BP reduction. However, magnitude of associations by LI is significantly higher than that by $\mathrm{CS}$, and there is some evidence that higher LI is associated with better BP outcomes. In a recent study from Japan, clinical benefit from adrenalectomy, as assessed according to the recent PASO consensus [12], was achieved in $61.7 \%$ of patients with an LI between 2 and 4 , in
$59.4 \%$ with an LI between 4 and 10, and in 77.8\% with an LI $>10$ ( $P<0.01$ for trend) [5]. In our study, the significant association of CS with clinical outcome disappeared after adjustment for other factors including LI (Supplemental Table S1). Similarly, Wolley et al. [8] showed that the significance of CS decreased when LI > 4 was applied as a cut-off. Therefore, the effect of CS may be reduced when the cut-off value used for LI is relatively high. In addition, the presence of aldosterone-producing cell clusters can exist at the contralateral adrenal gland, which attenuates the CS [23]. This may explain the less improvement in BP outcome in patients without $\mathrm{CS}$ than in those with it. Collectively, these results suggest that CS could be used complementarily to LI in predicting postoperative outcomes after unilateral adrenalectomy, particularly when the LI cut-off is relatively low.

Our study has several limitations. First, it is a retrospective and observational study by design, which is associated with a well-known risk of bias from residual confounders. As well, the BP measurement environment may not be the same. Second, we did not evaluate albuminuria as a renal outcome at pre- and post-adrenalectomy. Third, the number of cases without CS may be underestimated due to selection bias because there is a possibility that patients without CS were excluded for unilateral adrenalectomy. In previous retrospective studies, CS rates among patients who underwent adrenalectomy were around $82 \%$ to $97 \%$, similar to that of our study $[6,8,10,20]$. Fourth, we performed AVS only under ACTH stimulation. ACTH stimulation might increase success rate of selectivity, but reduce lateralization rate and identification of unilateral PA. Therefore, clinical implication of CS is necessary to be investigated in ACTH-unstimulated AVS in further study [24]. In addition, postoperative aldosterone suppression study was not undertaken in the present study. Hence, we did not fully assess the biochemical success according to the PASO study. Lastly, the SIT, which was used as a confirmatory test in our study, may exclude angiotensin-II-responsive APAs. Angiotensin-II-responsive APAs have been reported to comprise from $43 \%$ to $70 \%$ of cases [25].

Despite these limitations, the strength of our study is its relatively large number of patients from four tertiary hospitals compared to previous studies. To our best knowledge, this is one of the largest studies to analyze the clinical outcome of CS following adrenalectomy for PA. The clinical and biochemical outcome criteria of the PASO study were used in our study and DDD was assessed with standard ATC/DDD index [12]. Furthermore, the renal deterioration after adrenalectomy is not unusual complication and should be predicted with caution in patients with unilateral PA [16-18]. We showed the association be- 
tween CSI and renal impairment after adrenalectomy, which was not reported in previous studies. These results give an impression that it is important to be cautious of and manage postoperative GFR decline because of a high risk of incident CKD in patients $>50$ years with CSI $\leq 0.26$, especially.

In conclusion, $\mathrm{CS}$ may not predict postoperative clinical and biochemical outcomes in patients with unilateral aldosterone excess. Our results indicate that $\mathrm{CS}$ is associated with postsurgical deterioration of renal function. More attention should be paid to postoperative renal function in patients with unilateral PA when they have CSI $\leq 0.26$ at AVS before adrenalectomy, particularly in patients over 50 years of age.

\section{CONFLICTS OF INTEREST}

No potential conflict of interest relevant to this article was reported.

\section{ACKNOWLEDGMENTS}

This study was funded by the Asan Institute for Life Sciences, Seoul, Republic of Korea (Project No. 2019IP0862).

\section{AUTHOR CONTRIBUTIONS}

Conception or design: Y.S.Y., S.H.L., J.H.K., J.H.K., S.W.K. Acquisition, analysis, or interpretation of data: Y.S.Y., S.H.L., J.H.K., J.H.Y., J.H.L., S.Y.L., A.R.H., D.H.L., J.M.K., J.H.K., S.W.K. Drafting the work or revising: Y.S.Y., S.H.L., J.H.K., S.W.K. Final approval of the manuscript: Y.S.Y., S.H.L., J.H.K., J.H.Y., J.H.L., S.Y.L., A.R.H., D.H.L., J.M.K., J.H.K., S.W.K.

\section{ORCID}

Ye Seul Yang https://orcid.org/0000-0001-9682-6138

Seung Hun Lee https://orcid.org/0000-0003-0496-247X

Sang Wan Kim https://orcid.org/0000-0001-9561-9110

\section{REFERENCES}

1. Rossi GP, Bernini G, Caliumi C, Desideri G, Fabris B, Ferri $\mathrm{C}$, et al. A prospective study of the prevalence of primary aldosteronism in 1,125 hypertensive patients. J Am Coll Cardiol 2006;48:2293-300.

2. Kayser SC, Dekkers T, Groenewoud HJ, van der Wilt GJ, Carel Bakx J, van der Wel MC, et al. Study heterogeneity and estimation of prevalence of primary aldosteronism: a systematic review and meta-regression analysis. J Clin Endocrinol Metab 2016;101:2826-35.

3. Funder JW, Carey RM, Mantero F, Murad MH, Reincke M, Shibata $\mathrm{H}$, et al. The management of primary aldosteronism: case detection, diagnosis, and treatment: an endocrine society clinical practice guideline. J Clin Endocrinol Metab 2016;101:1889-916.

4. Monticone S, Viola A, Rossato D, Veglio F, Reincke M, Gomez-Sanchez C, et al. Adrenal vein sampling in primary aldosteronism: towards a standardised protocol. Lancet Diabetes Endocrinol 2015;3:296-303.

5. Umakoshi H, Tsuiki M, Yokomoto-Umakoshi M, Takeda Y, Takashi Y, Kurihara I, et al. Correlation between lateralization index of adrenal venous sampling and standardized outcome in primary aldosteronism. J Endocr Soc 2018;2:893902.

6. Tagawa M, Ghosn M, Wachtel H, Fraker D, Townsend RR, Trerotola S, et al. Lateralization index but not contralateral suppression at adrenal vein sampling predicts improvement in blood pressure after adrenalectomy for primary aldosteronism. J Hum Hypertens 2017;31:444-9.

7. Williams TA, Reincke M. Management of endocrine disease: diagnosis and management of primary aldosteronism: the Endocrine Society guideline 2016 revisited. Eur J Endocrinol 2018;179:R19-29.

8. Wolley MJ, Gordon RD, Ahmed AH, Stowasser M. Does contralateral suppression at adrenal venous sampling predict outcome following unilateral adrenalectomy for primary aldosteronism?: a retrospective study. J Clin Endocrinol Metab 2015;100:1477-84.

9. Umakoshi H, Tanase-Nakao K, Wada N, Ichijo T, Sone M, Inagaki $\mathrm{N}$, et al. Importance of contralateral aldosterone suppression during adrenal vein sampling in the subtype evaluation of primary aldosteronism. Clin Endocrinol (Oxf) 2015;83:462-7.

10. Monticone S, Satoh F, Viola A, Fischer E, Vonend O, Bernini G, et al. Aldosterone suppression on contralateral adrenal during adrenal vein sampling does not predict blood pressure response after adrenalectomy. J Clin Endocrinol Metab 2014;99:4158-66.

11. Shin SY, Park YR, Shin Y, Choi HJ, Park J, Lyu Y, et al. A de-identification method for bilingual clinical texts of various note types. J Korean Med Sci 2015;30:7-15.

12. Williams TA, Lenders JW, Mulatero P, Burrello J, Rottenkolber M, Adolf C, et al. Outcomes after adrenalectomy for 
unilateral primary aldosteronism: an international consensus on outcome measures and analysis of remission rates in an international cohort. Lancet Diabetes Endocrinol 2017;5: 689-99.

13. Hundemer GL, Curhan GC, Yozamp N, Wang M, Vaidya A. Renal outcomes in medically and surgically treated primary aldosteronism. Hypertension 2018;72:658-66.

14. Chen ZW, Hung CS, Wu VC, Lin YH; TAIPAI Study Group. Primary aldosteronism and cerebrovascular diseases. Endocrinol Metab (Seoul) 2018;33:429-34.

15. Sechi LA, Colussi G, Di Fabio A, Catena C. Cardiovascular and renal damage in primary aldosteronism: outcomes after treatment. Am J Hypertens 2010;23:1253-60.

16. Sechi LA, Novello M, Lapenna R, Baroselli S, Nadalini E, Colussi GL, et al. Long-term renal outcomes in patients with primary aldosteronism. JAMA 2006;295:2638-45.

17. Sechi LA, Di Fabio A, Bazzocchi M, Uzzau A, Catena C. Intrarenal hemodynamics in primary aldosteronism before and after treatment. J Clin Endocrinol Metab 2009;94:11917.

18. Park KS, Kim JH, Yang YS, Hong AR, Lee DH, Moon MK, et al. Outcomes analysis of surgical and medical treatments for patients with primary aldosteronism. Endocr J 2017;64: 623-32.

19. Park KS, Kim JH, Ku EJ, Hong AR, Moon MK, Choi SH, et al. Clinical risk factors of postoperative hyperkalemia af- ter adrenalectomy in patients with aldosterone-producing adenoma. Eur J Endocrinol 2015;172:725-31.

20. Shariq OA, Bancos I, Cronin PA, Farley DR, Richards ML, Thompson GB, et al. Contralateral suppression of aldosterone at adrenal venous sampling predicts hyperkalemia following adrenalectomy for primary aldosteronism. Surgery 2018;163:183-90.

21. Tahir A, McLaughlin K, Kline G. Severe hyperkalemia following adrenalectomy for aldosteronoma: prediction, pathogenesis and approach to clinical management: a case series. BMC Endocr Disord 2016;16:43.

22. Sukor N, Gordon RD, Ku YK, Jones M, Stowasser M. Role of unilateral adrenalectomy in bilateral primary aldosteronism: a 22-year single center experience. J Clin Endocrinol Metab 2009;94:2437-45.

23. Omata K, Satoh F, Morimoto R, Ito S, Yamazaki Y, Nakamura $\mathrm{Y}$, et al. Cellular and genetic causes of idiopathic hyperaldosteronism. Hypertension 2018;72:874-80.

24. Rossitto G, Amar L, Azizi M, Riester A, Reincke M, Degenhart $\mathrm{C}$, et al. Subtyping of primary aldosteronism in the avis2 study: assessment of selectivity and lateralization. J Clin Endocrinol Metab 2020;105:dgz017.

25. Rossi GP, Seccia TM, Pessina AC. A diagnostic algorithm: the holy grail of primary aldosteronism. Nat Rev Endocrinol 2011;7:697-9. 